\title{
ASSOCIATED AND PERSPECTIVE SIMPLEXES
}

\author{
BY
}

\section{LEON GER BER}

\begin{abstract}
A set of $n+1$ lines in $n$-space such that any $(n-2)$-dimensional flat which meets $n$ of the lines also meets the remaining line is said to be an associated set of lines. Two simplexes are associated if the joins of corresponding vertices are associated. A simple criterion is given for simplexes to be associated and an analogous one for simplexes to be perspective. These are used to give a brief proof of the following generalization of the theorem of Pappus.

Let $A$ and $B$ be $n$-simplexes and let $p$ be a permutation on the vertices of $B$. If $A$ and $B$ are associated (respectively perspective) and $A$ and $B p$ are associated (perspective) then $A$ and $B p^{k}$ are associated (perspective) for any integer $k$. Very short proofs are given of extensions to $n$-dimensions of many theorems from Neuberg's famous Memoir sur le Tétraèdre, such as: the altitudes of a simplex are associated.
\end{abstract}

1. Introduction. A set of $n+1$ lines in $n$-space having the property that any $(n-2)$-dimensional flat which meets $n$ of the lines meets the remaining line is said to be an associated set of lines (see [7], [2]). In 1884, in a famous Memoir [13], J. Neuberg established many of the properties of associated tetrahedra, that is, tetrahedra the joins of whose corresponding vertices are associated lines. In 1905 Berzolari [2] gave a necessary and sufficient condition for two $n$-simplexes to be associated, proved that the altitudes of an $n$-simplex are associated (which result was considered new when proved more than fifty years later [10]), and extended to $n$-dimensions both Neuberg's theorem on orthological tetrahedra and, in a very special way, Pappus' theorem on perspective triangles. In 1907 Neuberg [14] gave a much shorter proof of Berzolari's criterion, valid only in 3 space, and a number of applications.

In this paper we give a proof of Berzolari's criterion for associativity as well as a criterion for perspectivity which is very similar. In the sequel we give a large number of very short proofs of extensions of Neuberg's theorems to $n$-dimensions as well as the $n$-dimensional version of Pappus' theorem in its full generality.

Received by the editors August 13,1973.

AMS(MOS) subject classifications (1970). Primary 50B99; Secondary 50D05.

Key words and phrases. Associated lines, hyperbolic group of lines, perspective, simplex, orthological simplexes, polar simplex, Pappus theorem. 
Since the Neuberg Memoir is not easily accessible, we have included more accessible references as well.

A few remarks about associated lines may help orient the reader. In 2 space, given two distinct lines there is a single point which lies on both lines and any line through this point is associated with the given lines. In higher dimensions, then, associated lines may be thought of as a generalization of concurrent lines and many theorems of plane geometry can be extended to higher dimension in this way.

For $n=3$ there is a 1-parameter family of lines which meet three given skew lines. These form one set of rulings of a hyperboloid of one sheet if there is no plane parallel to the three given lines, and the rulings of a hyperbolic paraboloid if there is such a plane [9, pp. 14-15]. Any line, distinct from the given lines, of the 1-parameter family of conjugate rulings is associated with the given lines. This explains why some authors call a set of associated lines a hyperbolic set [2], [11]. In the plane, every set of three concurrent lines is the set of altitudes of some triangle, but in 3-space a set of four associated lines need not be the altitudes of some tetrahedron [3, p. 75].

For $n=4$, there is a 2-parameter family of planes which meet four given skew lines and in general there is a unique fifth line which meets these planes, i.e. is associated with the given lines [1, pp. 115-116]. For $n>4$ there is in general no line which meets the $(n-2)$-parameter family of $(n-2)$-flats each of which meets $n$ given lines, i.e. no line associated with $n$ given lines.

2. Notation and preliminaries. The setting for our theorems is Euclidean $n$ dimensional space augmented by an improper point (point at infinity) on each line which is defined to be the 1-dimensional vector space determined by pairs of points on the line. Any nonzero vector in this space is a representative of the improper point. The duality principle holds: in any true incidence statement we may replace "point" by "prime" $((n-1)$-flat) and "line" by "secundum" $((n-2)$-flat), providing we reverse any inclusions. Thus any $n+1$ secunda are said to be associated if any line which meets $n$ of the secunda also meets the remaining secundum, and associated secunda are a generalization of collinear points. It is clear that any correlation (a transformation that sends $k$-flats into $(n-1-k)$ flats and preserves incidence) sends a set of associated lines into a set of associated secunda and vice versa.

Let $I$ represent that set $\{0,1, \cdots, n\}, I(i)=I-\{i\}$, and $I^{\prime}=I(0)$, and let the ranges of $\Sigma, \Sigma^{(i)}, \Sigma^{\prime}$ be $I, I(i)$, and $I^{\prime}$ respectively. Throughout this paper $A$ will be a proper $n$-simplex with corresponding vertices and faces given by $A_{i}, \mathrm{~A}_{i}, i \in I$. In general, an upper case letter represents a point and a lower case boldface letter its position vector if it is proper or a representative if 
it is improper. Any point $B$ has an expression in the form $\mathbf{b}=\Sigma b_{i} \mathbf{a}_{i}$ where $s(B)=\Sigma b_{i}=1$ if $B$ is proper and $s(B)=0$ if $B$ is improper. If $B$ is proper the expression is unique, while if $B$ is improper there is a unique expression for each representative of $B$. The $b_{i}$ are called the weights (barycentric coordinates) of $B$ (with respect to $A$ ) and we write $B=\left(b_{0}, \cdots, b_{n}\right)=\left(b_{i}\right)$. The $b_{i}$ are sometimes called areal coordinates: If $c_{n}$ is the $n$-dimensional content (signed volume) function and $B_{i}$ is the simplex obtained from $A$ by replacing the vertex $A_{i}$ by $B$, then $b_{i}=c_{n}\left(B_{i}\right) / c_{n}(\mathrm{~A})$, for $i \in I$. Dually, the weights $\left(x_{i}\right)$ of the points $X$ of any prime $F$ satisfy a unique (to within a multiplicative constant) homogeneous equation $\Sigma u_{i} x_{i}=0$. The $u_{i}$ are called the weights of $F$ and we write $F=\left(u_{i}\right)$. Thus $A_{i}=\left(\delta_{i j}\right)$ where $\delta_{i j}$ is 1 if $j=i$ and 0 otherwise, and $(1,1, \cdots, 1)$ is (the weights of) the prime which consists of all improper points. The $u_{i}$ are sometimes called tangential coordinates: they are proportional to the lengths of parallel cevians from the $A_{i}$ to $F$. (A cevian of a simplex is a line passing through a vertex.)

If $B$ is a proper point, let $d_{i}$ be the distance of $B$ from $A_{i}$, positive if $B$ is on the same side of $A_{i}$ as $A_{i}$ and negative if $B$ is on the other side, $i \in I$. The $d_{i}$ are called the heights (absolute normal coordinates) of $B$. Let $\overrightarrow{A_{i} H_{i}}=\mathrm{h}_{i}$ be the altitude from $A_{i}$ and $h_{i}$ its length. If $B=\left(b_{i}\right)$, then $d_{i}=$ $b_{i} h_{i}$ in magnitude and sign. Let $v_{n}=\left|c_{n}\right|$ be the $n$-dimensional volume function, $v=v_{n}(A)$ and $f_{i}=v_{n-1}\left(A_{i}\right), i \in I$. Then $f_{i} h_{i}=n v$ and $f_{i} d_{i}=n v b_{i}$.

Finally, $B$ will denote an $n$-simplex, which may degenerate or have improper vertices, in the space of $A$ with corresponding vertices and faces given by $B_{i}, B_{i}, i \in I$. The matrix $\left(b_{i j}\right)$ will have for its $i$ th row the weights of $B_{i}$ $i \in I$. The equation of $B_{i}$ is $D_{i}=0$ where $D_{i}$ is the determinant of the matrix obtained from $\left(b_{i j}\right)$ by replacing row $i$ by $\left(x_{0}, \cdots, x_{n}\right)$. Thus the matrix of weights of the $B_{i}$ has as its elements the cofactors of $\left(b_{i j}\right)$.

3. Fundamental result. We now prove a simple theorem about concurrent cevians whose generalization to associated cevians will be our basic tool. Let us call the matrix $\left(b_{i j}\right)$ composite if there exist numbers $y_{i}, z_{i}, i \in I$, such that $b_{i j}=y_{i} z_{j}$ for $i \neq j$.

THEOREM 3.1. The cevians $A_{i} B_{i}, i \in I$, are concurrent if and only if the matrix $\left(b_{i j}\right)$ is composite.

Proof. The lines $A_{i} B_{i}$ concur at $P=\left(p_{j}\right)$ if and only if, for $i \in I$, $\mathrm{b}_{i}=u_{i} \mathrm{a}_{i}+w_{i} \mathrm{p}$ for some $u_{i} w_{i}$ where $u_{i}=1-w_{i},-w_{i}, 1$, or 0 according as both $P$ and $B_{i}, P$ only, $B_{i}$ only, or neither is proper. If the lines concur at $P$, then it is clear that $b_{i j}=w_{i} p_{j}$ for $i \neq j$. Conversely, suppose $b_{i j}=y_{i} z_{j}, i \neq j$. 
Suppose $\Sigma z_{i}=z \neq 0$. Then $b_{i j}=w_{i} p_{j}, i \neq j$, where $w_{i}=y_{i} z, p_{j}=z_{j} / z$ and $\Sigma p_{j}=1$. Let $s_{i}=s\left(B_{i}\right)$. Then

$$
b_{i i}=s_{i}-\sum_{j}^{(i)} b_{i j}=s_{i}-\sum_{j}^{(i)} w_{i} p_{j}=\left(s_{i}-w_{i}\right)+w_{i} p_{i}
$$

so $b_{i}=\left(s_{i}-w_{i}\right) a_{i}+w_{i} p$ where $P=\left(p_{i}\right)$. If $\Sigma z_{i}=0$, then

$$
b_{i i}=s_{i}-\sum_{j}^{(i)} y_{i} z_{j}=s_{i}+y_{i} z_{i}
$$

so $\mathrm{b}_{i}=s_{i} \mathrm{a}_{i}+y_{i} \mathrm{p}$ where $P=\left(z_{i}\right)$, which completes the proof.

We observe that if $\left(b_{i j}\right)$ is composite with constants $y_{i}, z_{i}, i \in I$, it follows that $\left(z_{i} / y_{i}\right) b_{i j}=\left(z_{j} / y_{j}\right) b_{j i}$. We shall call the matrix $\left(b_{i j}\right)$ semisymmetric if we can find numbers $x_{i}, i \in I$, not all zero, such that $x_{i} b_{i j}=x_{j} b_{i i}$ for $i$, $j \in I$. Let $M$ be the class of semisymmetric matrices. It follows that if a matrix $A \in M$, then its transpose $A^{T}$, the matrix obtained by multiplying a row or column by a nonzero constant, and the matrix of cofactors are all semisymmetric. The class of composite matrices is also closed under these operations. We are now ready for our basic tool ([2], [14, p. 299]).

THEOREM 3.2. The cevians $A_{i} B_{i}, i \in I$, are associated if and only if the matrix $\left(b_{i j}\right)$ is semisymmetric.

Proof. Suppose $\left(b_{i j}\right) \in M$ with multipliers $x_{i}, i \in I$. For $i=2, \cdots, n$, let $P_{i}$ lie on $A_{i} B_{i}$ so $\mathrm{p}_{i}=w_{i} \mathrm{a}_{i}+u_{i} \mathrm{~b}_{i}$ where $u_{i}=s\left(P_{i}\right)-w_{i}$. The secundum determined by the $P_{i}$ meets $A_{0} B_{0}$ if and only if $A_{0}, B_{0}$, and the $P_{i}$ lie in some prime, i.e.

$$
\begin{aligned}
0 & =\left|\begin{array}{rrrrr}
1 & 0 & 0 & \cdots & 0 \\
b_{00} & b_{01} & b_{02} & \cdots & b_{0 n} \\
u_{2} b_{20} & u_{2} b_{21} & u_{2} b_{22}+w_{2} & \cdots & u_{2} b_{2 n} \\
& & \cdots & & \\
u_{n} b_{n 0} & u_{n} b_{n 1} & u_{n} b_{n 2} & \cdots & u_{n} b_{n n}+w_{n}
\end{array}\right| \\
& =\left|\begin{array}{rrrr}
b_{01} & b_{02} & \cdots & \\
u_{2} b_{21} & u_{2} b_{22}+w_{2} & \cdots & b_{0 n} b_{2 n} \\
u_{n} b_{n 1} & u_{n} b_{n 2} & \cdots & u_{n} b_{n n}+w_{n}
\end{array}\right| .
\end{aligned}
$$


Multiplying row zero by $x_{0}$ and row $i$ by $x_{i} / u_{i} i=2, \cdots, n$, results in a determinant which is symmetric in the subscripts 0 and 1 , i.e. the secundum also meets $A_{1} B_{1}$. (If some $u_{i}$ is zero, first expand in terms of the corresponding row.)

To prove the converse, assume the cevians $A_{i} B_{i} i \in I$, are associated. Dividing the rows of the last determinant above by the $u_{i}, i=2, \cdots, n$, and setting $r_{i}=b_{i i}+w_{i} / u_{i}$ we see that

$$
\left|\begin{array}{lllll}
b_{01} & b_{02} & b_{03} & \cdots & b_{0 n} \\
b_{21} & r_{2} & b_{23} & \cdots & b_{2 n} \\
b_{31} & b_{32} & r_{3} & \cdots & b_{3 n} \\
& & \cdots & & \\
b_{n 1} & b_{n 2} & b_{n 3} & \cdots & r_{n}
\end{array}\right|=0
$$

implies

$$
\left|\begin{array}{ccccc}
b_{10} & b_{12} & b_{13} & \cdots & b_{1 n} \\
b_{20} & r_{2} & b_{23} & \cdots & b_{2 n} \\
b_{30} & b_{32} & r_{3} & \cdots & b_{3 n} \\
& & \cdots & & \\
b_{n 0} & b_{n 2} & b_{n 3} & \cdots & r_{n}
\end{array}\right|=0
$$

for all values of the $r_{i}$. As $P_{i}$ varies, $r_{i}$ takes on all real values. Thus as a polynomial in the $r_{i}$ the second is $b_{01} / b_{10}$ times the first. The respective coefficients of $r_{3} \cdots r_{n}$ are $-b_{21} b_{02}$ and $-b_{12} b_{20}$ so $\left(b_{02} / b_{20}\right) b_{21}=\left(b_{01} / b_{10}\right) b_{12}$. Working with the coefficients of the other terms of degree $n-2$ we get

$$
\left(b_{0 i} / b_{i 0}\right) b_{i 1}=\left(b_{01} / b_{10}\right) b_{1 i} \text { for } i=2,3, \cdots, n \text {. }
$$

Next, form the pair of determinant equations in which the special subscripts are 1 and 2 (instead of 0 and 1 ) and compare coefficients of $r_{0} r_{4} r_{5} \cdots r_{n}$ to get $\left(b_{12} / b_{21}\right) b_{23}=\left(b_{13} / b_{31}\right) b_{32}$. Since $\left(b_{02} / b_{20}\right) b_{21}=\left(b_{01} / b_{10}\right) b_{12}$. and $\left(b_{03} / b_{30}\right) b_{31}=\left(b_{01} / b_{10}\right) b_{13}$ we obtain

$$
\left(b_{02} / b_{20}\right) b_{23}=\left(b_{03} / b_{30}\right) b_{32} \text {. }
$$

In a similar fashion we get

$$
\left(b_{0 i} / b_{i 0}\right) b_{i j}=\left(b_{0 j} / b_{j 0}\right) b_{j i} \text { for all distinct } i, j \in I^{\prime} \text {. }
$$

If we take $x_{0}=1, x_{i}=b_{0 i} / b_{i 0}$ for $i \in I^{\prime}$, the proof is complete. 
Referring to the determinant of the direct proof we see that for each choice of parameters $w_{3}, \cdots, w_{n}$ we can choose $w_{2}$ so the determinant vanishes. This proves the assertion that there is an $(n-2)$-parameter family of secunda which meet $n$ of the lines.

Although we do not wish to discuss quadrics in this paper, the reader familiar with the subject will observe that an immediate consequence of Theorem 3.2 is the following ([2], [5], [14], [15]).

Corollary. The lines $A_{i} B_{i}, i \in I$, are associated if and only if $A$ and $B$ are polar with respect to some quadric.

This generalizes the known result for $n=2$ (Chasles' theorem and its converse [18, p. 125, Exercise 14]): The lines $A_{i} B_{\hat{i}} i=0,1,2$, are concurrent if and only if the triangles $A$ and $B$ are polar with respect to some conic. An independent proof of the "if" part of the corollary for the case of the sphere is given later as Theorem 6.3.

Following Neuberg $[14$, p. 304] (cf. [10, p. 409]) consider the polar of a simplex $A$ with respect to the absolute. In this case the polar of a face of $A$ is the improper point on a perpendicular to the face so the associated lines $A_{i} B_{i}$ of the Corollary are the altitudes of $A$. A metric proof that the altitudes of a simplex are associated is given later as Theorem 5.1.

The dual of Theorem 3.2 is ([2], [14]):

THEOREM 3.3. Let $B_{i}$ be a prime in the space of $A$ with weights $\left(b_{i j}\right)$, $i \in I$. Then the secunda $A_{i} \cap B_{i}$ are associated if and only if the matrix $\left(b_{i j}\right)$ is semisymmetric.

In the sequel we use these theorems to give short proofs of many theorems, some of which are new.

4. Desargues', Pappus', and other theorems. Each of the next two theorems reduces to Desargues' theorem for $n=2$. (For a different extension of Desargues' theorem see [11].)

THEOREM $4.1[18$, p. 54, Exercise 26]. Let $B$ be a simplex in the space of $A$. Then the lines $A_{i} B_{i} i \in I$, are concurrent at a point $P$ if and only if the secunda $A_{i} \cap B_{i} i \in I$, lie in a prime $F$. In either case we say $A$ and $B$ are perspective with $P$ and $F$ as center and axis of perspectivity.

This theorem is its own dual as is the following analogue ([2], cf. [3, pp. 119-120], [14]).

THEOREM 4.2. Let $B$ be a simplex in the space of $A$. Then the lines $A_{i} B_{i}, i \in I$, are associated if and only if the secunda $A_{i} \cap B_{i}, i \in I$, are associated. In either case we say the simplexes $A$ and $B$ are associated. 
Proof of TheOREMS 4.1 AND 4.2. The matrix of the weights of the $B_{i}$ is the matrix of cofactors of the weights of the $B_{i}$. Thus the hypothesis of each theorem is equivalent to the conclusion, via the remark preceding Theorem 3.2.

Our next result concerns the theorem of Pappus which is usually stated as:

If the six vertices of a hexagon lie alternately on two lines, then the three points of intersection of pairs of opposite sides are collinear.

However, the theorem can also be stated as follows [18, p. 100]:

If triangle $A_{0} A_{1} A_{2}$ is perspective with $B_{0} B_{1} B_{2}$ and with $B_{1} B_{2} B_{0}$, then it is also perspective with $B_{2} B_{0} B_{1}$.

In this form we can extend Pappus' theorem to $n$-space in its full generality (cf. [2]) in two ways. In each, $p$ is a permutation on $I$, ip denotes the image of $i$ under $p$, and $B p$ is the simplex with vertices $B_{0 p}, B_{1 p}, \cdots, B_{n p}$.

THEOREM 4.3. If $A$ and $B$ are perspective and $A$ and $B p$ are perspective, then $A$ and $B p^{k}$ are perspective for every integer $k$

THEOREM 4.4. If $A$ and $B$ are associated and $A$ and $B p$ are associated, then $A$ and $B p^{k}$ are associated for every integer $k$.

To prove Theorem 4.4 we prove the equivalent proposition: Let $q$ be the inverse of $p$. If $A$ and $B q$ are associated and $A$ and $B$ are associated, then $A$ and $B p$ are associated.

Let the matrix (of the weights of the vertices) of simplex $B$ be $\left(b_{i j}\right)$. By hypothesis, we can find nonzero numbers $x_{i}, i \in I$, such that $x_{i} b_{i j}=x_{j} b_{j i}$, and the matrix of $B q=\left(b_{i q, j}\right) \in M$, the class of semisymmetric matrices. Applying $p$ to the row and column subscripts of the latter (equivalent to relabelling the corresponding vertices of $A$ and $B q)$ we get $\left(b_{i, j p}\right) \in M$. Its transpose $\left(b_{j, i p}\right) \in M$ and so $\left(x_{j} b_{j, i p}\right) \in M$. The last matrix equals $\left(x_{i p} b_{i p, j}\right)$ so $\left(b_{i p, j}\right) \in M$ and this is the matrix of $B p$. To prove Theorem 4.3 replace "associated" and " semisymmetric" by "perspective." and "composite" in the above proof.

The next three theorems are easy corollaries of Theorem 3.2. $P^{\prime}$ is called the isotomic (respectively isogonal) conjugate of the point $P$ with respect to $A$ if the weights (heights) of $P^{\prime}$ are inversely proportional to those of $P$. Thus $[14$, p. 301]:

THEOREM 4.5 (cf. [3, p. 139] and [13, p. 28]). Let $B_{i}^{\prime}$ denote the isotomic conjugate of $B_{i}$ with respect to $A$. Then the cevians $A_{i} B_{i} i \in I$, are associated (concurrent) if and only if the cevians $A_{i} B_{i}^{\prime} i \in I$, are associated (concurrent).

THEOREM $4.6\left[13\right.$, p. 28]. Let $B_{i}^{\prime}$ denote the isogonal conjugate of $B_{i}$ 
with respect to $A$. Then the cevians $A_{i} B_{i}, i \in I$, are associated (concurrent). if and only if the cevians $A_{i} B_{i}^{\prime}, i \in I$, are associated (concurrent).

Dually, we say that $F^{\prime}$ is the reciprocal transversal of the prime $F$ if its weights are inversely proportional to those of $F$. Thus we have

THEOREM 4.7. Let $B_{i}^{\prime}$ be the reciprocal transversal of $B_{i}$ with respect to A. Then the secunda $A_{i} \cap B_{i}, i \in I$, are associated (coprime) if and only if the secunda $A_{i} \cap B_{i}$ are associated (coprime).

The point $P$ and prime $F$ are said to be pole and polar with respect to A if the weights of $P$ are inversely proportional to those of $F$. (In this case $F$ is the axis of perspectivity for $A$ and the pedal simplex of $P$ with respect to A.) Thus

THEOREM 4.8. Let $B_{i}$ and $F_{i}$ be pole and polar with respect to $A, i \in I$. Then the cevians $A_{i} B_{i}$ are associated (concurrent) if and only if the secunda $A_{i} \cap F_{i}$ are associated (coprime).

5. Perpendiculars. Our first theorem is the recently rediscovered result on altitudes [2], [7], [10], [13, p. 23], [14, p. 304].

THEOREM 5.1. The altitudes of a simplex are associated.

Proof. $H_{i}$, the foot of the altitude from $A_{i}$, is the orthogonal projection of $A_{i}$ on $A_{i}$. Thus its $j$ th weight with respect to $A_{i}$, and hence with respect to $A$, is the $(n-1)$-content of the projection of $A_{j}$ on $A_{i}$ divided by the $(n-1)$ content of $A_{i}, j \in I(i)$, i.e. $n_{i i}=0$ and $h_{i j}=f_{j} f_{i}^{-1} \cos \left(A_{i}, A_{j}\right)$ for $j \in I(i)$. Thus $f_{i}^{2} h_{i j}=f_{i} f_{j} \cos \left(A_{i}, A_{j}\right)=f_{i}^{2} n_{j i}$.

COROLlARY (cf. [13, p. 29]). The altitudes concur if and only if the matrix $\left(\cos \left(A_{i}, A_{j}\right)\right)$ is composite.

COROLlaRy [3, p.76, Excercise 5]. The perpendiculars to the prime faces of a simplex at their centroids are associated.

Proof. The dilatation with center at the centroid and constant $-1 / n$ sends the altitudes into these perpendiculars.

The case of concurrency (for $n=3$ ) in the next theorem is due to Steiner [3, pp. 173-174, p. 342] in which case $A$ and $B$ are said to be mutually orthological. The associated case (for $n=3$ ) is due to Neuberg [13, p. 27], in which case $A$ and $B$ are called skew orthological.

THEOREM 5.2. Let $B$ be a proper simplex in the space of $A$. If the perpendiculars from $A_{i}$ to $B_{i}, i \in I$, are associated (concurrent), then the perpen- 
diculars from $B_{i}$ to $A_{i}, i \in I$, are associated (concurrent).

Proof. Let $P_{i}$ be the foot of the perpendicular from $A_{i}$ to $B_{i}$ and $p_{i}$ its length, and $c_{i}^{j}$ the cosine of the angle between $A_{j}$ and $B_{i}$. It is easy to see that for $j \in I(i)$ the signed distance of $P_{i}$ from $A_{j}$ is $p_{i} c_{i}^{j}$ so the $j$ th weight of $P_{i}$ with respect to $A$ is $(1 / n) f_{j} p_{i} c_{i}^{j}$. It follows that $\left(c_{i}^{j}\right)$ is semisymmetric (composite) and hence $\left(c_{j}^{i}\right)$ is semisymmetric (composite). The conclusion follows by expressing the weights of the feet of the perpendiculars from $B_{i}$ to $A_{i}$ with respect to $B$.

6. Spheres. Let $S(S, r)$ be the sphere with center $S$ and radius $r$. Two proper points $X$ and $P$ are conjugate with respect to $S$ if, with $S$ as origin, $\mathbf{p} \cdot \mathbf{x}=r^{2}$; if either $X$ or $P$ is improper, the condition is $\mathbf{p} \cdot \mathbf{x}=0$. Thus the locus of points conjugate to a $k$-flat $F$ is an $(n-1-k)$-flat $F^{\prime}$, completely perpendicular to $F$, called the polar of $F$ and their unique common perpendicular passes through $S$. In particular, the polar of $S$ is the improper prime and if $F$ contains $S$ then $F^{\prime}$ is entirely improper. The transformation that sends a flat into its polar is a correlation, called a polarity, hence

THEOREM 6.1. If $F_{i}, i \in I$, is an associated set of lines, then $F_{i}^{\prime}, i \in I$, is an associated set of secunda.

The simplex $A^{\prime \prime}$ which has for its vertices $A_{i}^{\prime}$ and for its faces $A_{i}^{\prime}, i \in I$, is called the polar simplex of $A$.

THEOREM 6.2. If $A$ and $B$ are associated simplexes, so are $A$ " and $B "$.

Proof. The hypothesis is that the lines $A_{i} B_{i}, i \in I$, are associated. By Theorem 6.1, the secunda $A_{i}^{\prime} \cap B_{i}^{\prime}=A_{i}^{\prime \prime} \cap B_{i}^{\prime \prime}, i \in I$, are associated. The conclusion follows from Theorem 4.2.

The preceding theorem states that a polarity preserves an existing associative relation. The next theorem shows that a polarity generates an associative relation [2], [5], [14], [15].

THEOREM 6.3. A simplex and its polar simplex are associated.

Proof. Let $A$ be the given simplex and let $B_{i}=\left(b_{i j}\right)$ be the polar of $A_{i}$ with respect to $S(S, r)$. Let $s_{i}=s\left(A_{i}\right)$ and let $X=\left(x_{i}\right)$ be a proper point of $B_{i}$. The equation of $B_{i}$ is

$$
0=\mathbf{a}_{i} \cdot \mathbf{x}-s_{i} r^{2}=\mathbf{a}_{i} \cdot \sum x_{j} \mathbf{a}_{j}-s_{i} r^{2} \sum s_{j} x_{j}=\sum\left(\mathbf{a}_{i} \cdot \mathbf{a}_{j}-s_{i} s_{j} r^{2}\right) x_{j}
$$

so $b_{i j}=a_{i} \cdot a_{j}-s_{i} s_{j} r^{2}=b_{j i}$. The result follows from Theorems 3.3 and 4.2.

Applying the result to the special case $r=0$ we get 
THEOREM 6.4 [3, p. 244, EXERCISE 36], [16]. Let $S$ be any point in the space of $A$ and let $F_{i}$ be a prime through $S$ perpendicular to $S A_{i}, i \in I$. Then the secunda $F_{i} \cap A_{i}$ are associated, $i \in I$.

The tangent primes to the circumsphere of a simplex at its vertices are the polars of the vertices with respect to the circumsphere and form the tangential simplex. Thus [3, pp. 117-118], [13, p. 24]

THEOREM 6.5. A simplex and its tangential simplex are associated.

For $n=2$ we recognize this theorem as a special case of Brianchon's theorem. Reversing the roles of the two simplexes in the previous theorem we get $[3$, p. 118$],[13$, p. 24$]$ :

THEOREM 6.6. Let $B_{i}$ be the point of contact of the inscribed sphere of $A$ with $A_{i}, i \in I$. Then $A$ and $B$ are associated.

THEOREM $6.7[13$, p. 23], [17, p. 61]. The lines joining the vertices of a simplex to the incenters of the opposite faces are associated.

Proof. Let $I_{i}=\left(w_{i j}\right)$ be the incenter of $A_{i}$. Let $A_{i j}=A_{i} \cap A_{j}$, let $f_{i j}=v_{n-2}\left(A_{i j}\right)$ and let $k_{i}=\Sigma_{j}^{(i)} f_{i j}$. Then $w_{i j}=f_{i j} / k_{i}\left[6\right.$, p. 453] so $k_{i} w_{i j}=$ $f_{i j}=k_{j} w_{i j}$.

We remark in passing that the lines joining the vertices of a simplex to the circumcenters of the opposite faces are not in general associated.

We next prove a generalization of the theorem that the bisectors of the angles of a triangle concur. A corner with vertex $A_{i}$, the analogue of an angle, is what remains of the simplex $A$ when the face $A_{i}$ is deleted. An isoclinal prime of a corner is a prime which cuts equal segments on the edges of the corner; all such primes are related by a dilatation with center $\boldsymbol{A}_{\boldsymbol{i}}$. A centroidal line of a corner is a line which joins the vertex to the centroid of the $(n-1)$-simplex determined by an isoclinal prime. The centroidal lines of a triangle are the angle bisectors. Let $|A B|$ denote the length of segment $A B$. An $n$-simplex $A$ is said to be isodynamic if there exist positive numbers $t_{i}, i \in I$, such that $\left|A_{i} A_{j}\right|=\dot{t}_{i} t_{j}, i \neq j$. (A full discussion of isodynamic $n$-simplexes for $n=3$ will be found in [3, pp. 314-330] and [17].) We have the following result. (The associated case is [3, p. 140, Example 2].)

THEOREM 6.8.'The centroidal lines of a simplex are associated; they concur if and only if the simplex is isodynamic.

Proof. For the vertex $A_{i}$ choose $D_{j}$ on $A_{i} A_{j}$ so that $\left|A_{i} D_{j}\right|=c_{i}, j \in I(i)$, so $\overrightarrow{A_{i} D_{j}}=c_{i}\left|A_{i} A_{j}\right|^{-1} \overrightarrow{A_{i} A_{j}}$. The centroid $B_{i}$ of the isoclinal prime determined by the $D_{j}$ is given by 


$$
\overrightarrow{A_{i} B_{i}}=c_{i} n^{-1} \sum_{j}^{(i)}\left|A_{i} A_{j}\right|^{-1}{\overrightarrow{A_{i}}}_{j}
$$

If we choose $c_{i}$ so that $c_{i}^{-1}=n^{-1} \Sigma_{j}^{(i)}\left|A_{i} A_{j}\right|^{-1}$, the expression on the right is independent of the origin and defines $B_{i}$ as a point of $A_{i}$ with weights $b_{i j}=$ $c_{i} n^{-1}\left|A_{i} A_{j}\right|^{-1}, j \neq i$. Thus $c_{i}^{-1} b_{i j}=n^{-1}\left|A_{i} A_{j}\right|^{-1}=c_{j}^{-1} b_{j i}$ and the lines are associated.

The lines will concur if and only if there exist numbers $x_{i}, y_{i}, i \in I$, such that $x_{i} y_{j}=c_{i} n^{-1}\left|A_{i} A_{j}\right|^{-1}$ for $i \neq j$. Then

$$
\left|A_{i} A_{j}\right|=\left|A_{i} A_{j}\right|^{1 / 2}\left|A_{j} A_{i}\right|^{1 / 2}=\left(n c_{i}^{-1} x_{i}^{-1} y_{i}^{-1}\right)^{1 / 2}\left(n c_{j}^{-1} x_{j}^{-1} y_{j}^{-1}\right)^{1 / 2}=t_{i} t_{j},
$$

so the simplex is isodynamic and conversely.

Let a prime meet the edges $A_{0} A_{i}$ of $A$ in $B_{i}, i \in I^{\prime}$. If the $2 n$ points $A_{i}, B_{i}, i \in I^{\prime}$ are cospherical, then $A_{0}$ and $B_{0}$ are said to be antiparallel sections of the corner at $A_{0}$. In this case $B_{0}$ is parallel to the prime tangent to the circumsphere at $A_{0}$ and conversely.

THEOREM 6.9 [3, p. 288, Exercise 6], [13, p. 30]. The lines joining the vertices of a simplex to the centroids of sections antiparallel to the corresponding faces are associated, and concur if and only if the simplex is isodynamic.

Proof. Let $p_{0}$ be the power of $A_{0}$ with respect to the sphere through $A_{j}$ and $B_{j}, j \in I^{\prime}$. Then $\overrightarrow{A_{0} B_{j}}=p_{0} \mid A_{0} A_{j} \Gamma^{2} \overrightarrow{A_{0} A_{j}}, j \in I^{\prime}$, so the weights of the centroid $M_{0}$ of this section are given by $m_{0 j}=n^{-1} p_{0} \mid A_{0} A_{j} \Gamma^{-2}, j \in I^{\prime}$. Similarly, $M_{i}=\left(m_{i j}\right)$ with $m_{i j}=n^{-1} p_{i}\left|A_{i} A_{j}\right|^{-2}, j \in I(i)$. Since $p_{i} \neq 0, p_{i}^{-1} m_{i j}=$ $n^{-1}\left|A_{i} A_{j}\right|^{-2}=p_{j}^{-1} m_{j i}$ and the lines are associated. The case of concurrency is handled as in the preceding theorem.

7. The first Lemoine point of a simplex. Since there does not seem to be any discussion of this point in English (cf. [13], [17, pp. 147-149] for $n=3$, and [8] for the general case) we prove some preliminary results. We recall that $f_{i}=v_{n-1}\left(A_{i}\right)$.

THEOREM 7.1. If $P$ is a proper point in the space of $A$ with heights $\left(d_{i}\right)$ then $\Sigma d_{i}^{2} \geqslant n^{2} v^{2} / \Sigma f_{i}^{2}$. The minimum is attained at a unique point $K=\left(w_{i}\right)$ called the first Lemoine point of A where $w_{i}=f_{i}^{2} / \Sigma f_{i}^{2}$.

PROOF. An application of the Schwarz inequality yields:

$$
\sum d_{i}^{2}=n^{2} v^{2} \sum\left(w_{i} / f_{i}\right)^{2} \geqslant n^{2} v^{2}\left[\sum\left(w_{i} / f_{i}\right) f_{i}\right]^{2} / \sum f_{i}^{2}=n^{2} v^{2} / \sum f_{i}^{2} .
$$

Equality is attained if and only if $\left(w_{i} / f_{i}\right)$ and $\left(f_{i}\right)$ are proportional. Since $\Sigma w_{i}=1$ we have the result. 
THEOREM 7.2 (cf. [17, pp. 27-28]). There are, in general, $n$ primes which divide $n+1$ given ordered line segments in the same ratio.

Proof. Let the segments be $A_{i} B_{i}, i \in I$. Let $B_{i}=\left(b_{i j}\right)$ with respect to $A$ and let $\mathbf{p}_{i}=(1-r) \mathbf{a}_{i}+r \mathbf{b}_{i}$. The $P_{i}$ lie in a prime if and only if $\operatorname{det}\left(p_{i j}\right)=0$. Add each column to the first to get a column of 1's. Expand by the first column to get a polynomial of degree $n$ in $r$.

THEOREM 7.3 (cf. [17, pp. 148-149]). Any prime which divides the altitudes of a simplex in the same ratio contains the first Lemoine point.

Proof. Let the point $B_{i}$ lie on the altitude $A_{i} H_{i}$ so that $\overrightarrow{A_{i} B_{i}}=r \overrightarrow{A_{i} H_{i}}$, $i \in I$. Define the point $P$ by $\left(\Sigma f_{i}^{2}\right) p=\Sigma f_{i}^{2} b_{i}$ so that if the $B_{i}$ are coprime then $P$ lies in this prime. Let ' denote projection on $A_{0}$. Then

$$
\begin{aligned}
& \left(\Sigma f_{i}^{2}\right)\left|P P^{\prime}\right|=\sum f_{i}^{2}\left|B_{i} B_{i}^{\prime}\right|=(1-r) f_{0}^{2}\left|A_{0} H_{0}\right|+r \sum_{i}^{\prime} f_{i}^{2}\left|H_{i} H_{i}^{\prime}\right| \\
& =(1-r) f_{0}^{2}\left|A_{0} H_{0}\right|+r \sum^{\prime} f_{i}^{2}\left|A_{i} H_{i}\right| \cos \left(\mathrm{A}_{i}, \mathrm{~A}_{0}\right) \\
& =n v\left[(1-r) f_{0}+r \sum^{\prime} f_{i} \cos \left(A_{i}, A_{0}\right)\right]=r n v f_{0}
\end{aligned}
$$

and similarly for the other normal coordinates of $P$. Thus $P=K$.

THEOREM 7.4 (cf. [13, p. 23]). The lines joining the vertices of a simplex to the first Lemoine points of the opposite faces are associated.

Proof. Replace " $f_{i j}$ " by " $f_{i j}^{2}$ " in the proof of Theorem 6.7 .

Thanks are due to H. S. M. Coxeter for encouragement and for references [2] and [15].

\section{REFERENCES}

1. H. F. Baker, Principles of geometry. Vol. IV, Cambridge Univ. Press, New York, 1940.

2. L. Berzolari, Sui sistemi di $n+1$ rette dello spazio ad $n$ dimensioni situati in posizione di Schläfli, Rend. Circ. Mat. Palermo (2) 20 (1905), 229-247.

3. N. A. Court, Modern pure solid geometry, 2nd ed., Chelsea, New York, 1964.

4. H. S. M. Coxeter, Introduction to geometry, Wiley, New York, 1961. MR 23 \#A1251.

5. H. S. M. Coxeter and J. A. Todd, Solution of advanced problem 4079, Amer. Math. Monthly 51 (1944), 599-600.

6. L. Gerber, Spheres tangent to all the faces of a simplex, J. Combinatorial Theory 12 (1972), 453-456. MR $45 \# 7607$.

7. - The altitudes of a simplex are associated, Math. Mag. 46 (1973), 155-157.

8. H. Hanani, On a point of minimum sum of distances-squares from the faces of a simplex, Riveon Lematematika 7 (1954), 10-12. MR 15, 451. (Hebrew) 
9. D. Hilbert and S. Cohn-Vossen, Geometry and the imagination, Springer-Berlin, 1932; English transl., Chelsea, New York, 1952. MR 13, 766.

10. S. R. Mandan, Altitudes of a general n-simplex, J. Austral. Math. Soc. 5 (1965), 409-41 5. MR $32 \# 6277$.

11. - Desargues' theorem in n-space, J. Austral. Math. Soc. 1 (1959/60), 311 31 8. MR 23 \#A 4045.

12. - Isodynamic \& isogonic simplexes, Ann. Mat. Pura Appl. (4) 53 (1961), 45-55. MR 23 \#A 1253.

13. J. Neuberg, Memoir sur le tétraèdre, Académie Royale des Sciences des lettres et des beaux-arts de Belgique, Mémoires couronnés 37 (1884), 1-72.

14. - _ Über hyperboloidische Würfe, Arch. Math. Phys. (3) 12 (1907), 297-305.

15. L. Schläfli, Erweiterung des Satzes, das zwei polare Dreiecke perspectivisch liegen, auf eine beliebige Zahl von Dimensionen, J. Reine Angew. Math. 65 (1866), 189-197 (= Gesammelte mathematische A bhandlungen. Bd. III, Verlag Birkhäuser, Basel, 1956, pp. 9-20. MR 17, 814).

16. H. M. Taylor, Solution of question 15886, Educational Times Reprints (2) 10 (1906), 48.

17. V. Thébault, Parmi les belles figures de la géométrie dans l'espace, Librairie Vuibert, Paris, 1955. MR 16, 737.

18. O. Veblen and W. J. Young, Projective geometry, Blaisdell, New York, 1938.

DEPARTMENT OF MATHEMATICS AND COMPUTER SCIENCE, ST. JOHN'S UNIVERSITY, JAMAICA, NEW YORK 11439 